\title{
A technique for laparoscopic cystic duct cholangiography
}

Cholangiography may be indicated during the course of laparoscopic cholycystectomy. Many catheters for and techniques of performing cystic duct cholangiography have been developed. Catheters with balloons have the advantage of preventing leaks during dye injection, but balloon inflation may often result in displacement of the balloon either into the common bile duct or out of the biliary tree altogether, resulting in unsatisfactory studies. Catheters without balloons, on the other hand, are prone to leak contrast during injection, again resulting in poor X-rays. We have developed a technique for safe, rapid, and accurate cystic duct cholangiography without leakage of contrast.

Following identification and isolation of the cystic duct, Hartmann's pouch is elevated with a grasper via the midsubcostal port, exposing the entire length of the cystic duct. A clip is placed as high as possible on the cystic duct, and an anterior incision is made into the duct. The cystic duct is then cannulated via the lateral subcostal port with an Americath (American Catheter) cholangiogram catheter, with the first hashmark just inside the duct. The operator places the endoclip applier around the cystic duct and begins to close the clip while the assistant continuously injects saline into the catheter. The operator continues to close the clip until it is just tight enough to prevent further injection of contrast by the assistant. The operator releases the clip onto the duct, with the assistant still trying to inject. It has been our experience that the clip will be tight enough to prevent leaks but loose enough to permit flow of contrast. Cholangiography may then be performed without incident in the usual way. At its conclusion, the clip and catheter are removed, and the cystic duct may be clipped and divided. Alternatively, if the cholangiogram reveals choledocholithiasis, the common bile duct may be explored via the cystic duct or directly.

In summary, cholangiography is often indicated during laparoscopic cholecystectomy. A simple technique for leakproof cystic duct cholangiography is described. This procedure provides an effective means for obtaining high-quality, accurate cholangiograms without leakage of contrast or catheter displacement.

\section{H. J. Winter \\ C. J. Butler}

2301 Evesham Ave., Suite 504

Voorhees, NJ 08043

USA 